\title{
Psychische
}

\section{Begleiterkrankungen nach Schlaganfall}

\author{
Bernhard Elsner und Jan Mehrholz
}

\begin{abstract}
Einer von drei Menschen erfährt im Laufe seines Lebens eine Demenz, einen Schlaganfall oder beides. Neben Menschen mit klinisch prominenten demenziellen Syndromen interessieren sich Forschung und Gesundheitspolitik seit den letzten Jahren zunehmend für Menschen mit kognitiven Einschränkungen nach Schlaganfall. Psychische Begleiterkrankungen nach Schlaganfall sind häufig. Die kognitiven Einschränkungen genügen zwar in der Regel nicht den Kriterien einer klinischen Diagnose, beeinträchtigen aber erheblich die Lebensqualität und können den Therapieverlauf gravierend negativ beeinflussen.
\end{abstract}

So nimmt die Fragestellung nach der besten Möglichkeit zur Verbesserung der Kognition nach Schlaganfall Platz 1 in den Top-Ten-Prioritäten der Schlaganfallforschung ein [22]. Jedes Jahr erleiden 15 Millionen Menschen weltweit einen Schlaganfall [16]. In Deutschland sind jedes Jahr ca. 196000 Menschen erstmalig und 66000 zum wiederholten Male von einem Schlaganfall betroffen [7].

Unter der Annahme, dass die zukünftige Anzahl an Neuerkrankungen mindestens gleich bleibt, dürfen wir davon ausgehen, dass durch eine veränderte Demografie, die gestiegene Lebenserwartung und eine höhere Überlebensrate nach Schlaganfall die Anzahl von Menschen mit psychischen Begleiterkrankungen nach Schlaganfall weiter zunehmen wird [19].

\section{Arten psychischer Begleiterkrankungen}

Häufige Arten von psychischen Begleiterkrankungen nach Schlaganfall sind Demenz (post-stroke cognitive impairment, PSCl, oder auch post-stroke dementia, PSD), Depression (post-stroke depression, PSD) und Delir. Bisher gibt es zwar einige Klassifikationen, diagnostische Kriterien und beschreibende Syndrome, die benutzt werden können, um PSCl zu klassifizieren, jedoch fehlt es an einer breit akzeptierten und harmonisierten Terminologie.

Definitionen Vereinfacht gesagt beschreibt PSCI jeglichen kognitiven Verfall seit dem Eintreten eines Schlaganfalls [19]. PSCI ist eine Hauptuntergruppe vaskulär-kognitiver Einschränkungen (vascular cognitive impairment, $\mathrm{VCl}$, A Abb. 2).). Sie ist jedoch nicht deckungsgleich mit

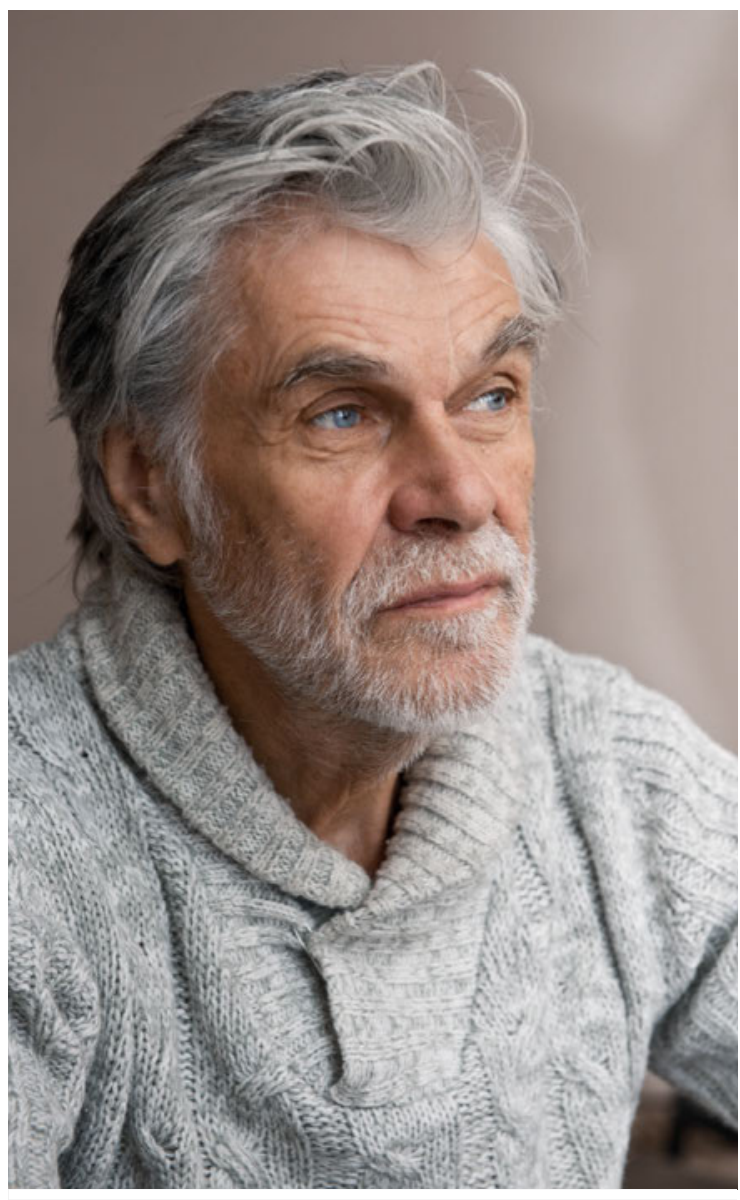

- Abb. 1 Häufig treten nach Schlaganfall psychische Begleiterkrankungen auf. (Quelle: Jonas Glaubitz/stock. adobe.com; nachgestellte Situation) 


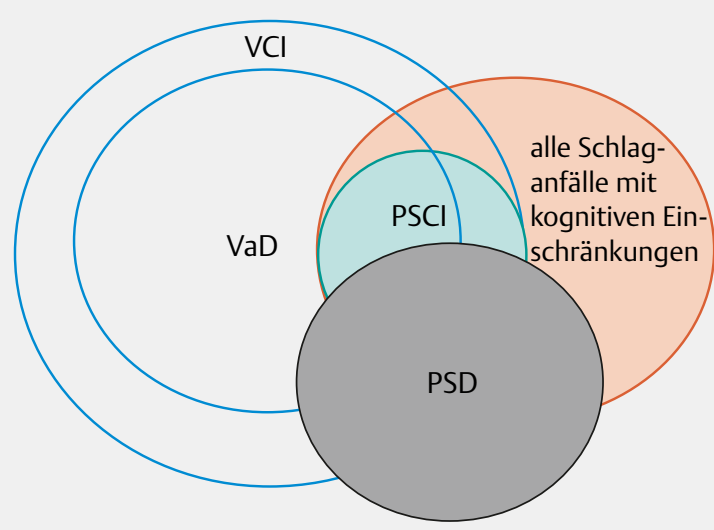

- Abb. 2 Venn-Diagramm zur Veranschaulichung der Abgrenzung der Konstrukte, um psychische Begleiterkrankungen nach Schlaganfall zu definieren.

VCl: vaskuläre kognitive Einschränkung (vascular cognitive impairment), VaD: vaskuläre Demenz (vascular dementia), PSCl: kognitive Einschränkung nach Schlaganfall (post-stroke cognitive impairment), PSD: Depression nach Schlaganfall (post-stroke depression). (Quelle: Grafik nach Mijajlovic et al. [19]; Umsetzung: Thieme Gruppe)

dem Begriff der vaskulären Demenz (vascular dementia, $\mathrm{VaD}$ ), da Letztere ein Konzept ist, das multiple kortikale oder subkortikale Infarkte, bedeutende Einzelinfarkte, nicht infarktbedingte Läsionen der weißen Substanz, Hirnblutungen und Hypoperfusion als Ursachen des kognitiven Abbaus einschließt.

$\mathrm{VaD}$ kann als eine Subgruppe der $\mathrm{VCl}$ angesehen werden, die eine voll entwickelte Demenz nach einem sicher identifizierten vaskulären Ereignis darstellt. Eine PSCI kann somit bereits vor dem Schlaganfall aufgrund einer VCI oder VaS bestanden haben oder aber auch erst durch den Schlaganfall entstanden sein. Einen Überblick zur Differenzierung der verschiedenen Definitionen bietet $>$ Abb. 3 [19].

Psychische Erkrankungen Menschen nach einem Schlaganfall können eine Vielzahl von psychischen Erkrankungen erfahren, die eher spezifisch für eine Hirnverletzung (organisch bedingte) als für eine (unipolare) Depression nach Standardkriterien (depressive Episode) sind [6]. So kann es nach Schlaganfall häufig zu z. B. emotionaler Verflachung, emotionaler Labilität, Enthemmung, krankhaftem Nichterkennen der offensichtlichen Schädigung durch den Schlaganfall (Anosognosie) und Schwierigkeiten beim emotionalen Ausdruck mittels Sprache (Aprosodie) kommen [6].

Delirium Das Syndrom des Deliriums ist eine häufige, vorübergehende Begleiterscheinung einer akuten Erkrankung, die klinisch gestellt wird [8]. Es ist gekennzeichnet durch ein plötzlich verändertes Bewusstseinsniveau mit einem wechselhaften Verlauf in der Orientierung, im Gedächtnis, im Denken oder Verhalten [24]. Typisch ist der plötzliche Beginn mit häufig nächtlicher Verschlechterung. Diese Diagnose wird eher selten gestellt und wenn doch, dann zumeist im Zusammenhang mit einer psych- iatrischen Vorerkrankung. Die Ätiologie ist häufig multifaktoriell [8].

\section{Zahlen, Daten, Fakten}

Nach Schlaganfall leidet jeder Vierte unter kognitiven Einschränkungen, jeder Dritte unter einer Depression und jeder Sechste unter einem Delir.

Eine Metaanalyse, die im Jahr 2009 in Lancet Neurology erschien, schloss zur Schätzung der Inzidenz von PSCI 22 krankenhaus- und acht populationsbasierte Kohortenstudien mit insgesamt 7511 Patienten ein [21]. Es zeigte sich, dass im Mittel einer von vier Menschen nach erstmaligem Schlaganfall positiv auf PSCI getestet wurde. Jeder zehnte von ihnen hatte bereits vor Eintreten des Schlaganfalls kognitive Einschränkungen. Bei einem Schlaganfall-Reinfarkt ist die Inzidenz höher. Insgesamt schwankt sie somit zwischen 7 und $41 \%$.

In einer Metaanalyse von 61 Beobachtungsstudien mit mehr als 25000 Teilnehmern aus dem Jahr 2014 zeigte sich, dass nach einem Schlaganfall im Mittel einer von drei Betroffenen eine Depression entwickelt [11]. Diese Inzidenz schwankte geringfügig je nach Zeitraum und Setting: Die höchste Inzidenz war kurz nach dem Schlaganfall und im Rehabilitationsbereich zu verzeichnen.

Eine Metaanalyse, die im Jahr 2012 in Stroke erschien, zeigte, dass die Wahrscheinlichkeit, nach einem Schlaganfall ein Delir zu entwickeln, fast doppelt so hoch ist wie auf einer durchschnittlichen internistischen Station (13-48\% versus $10-25 \%$ ) [24].

\section{Screenings}

Es gibt gute Screenings, jedoch werden sie häufig nicht genutzt.

Bei der Diagnostik neuropsychologischer Störungen sind zwei Ansätze weit verbreitet: das pauschale Screening aller Patienten mit Schlaganfall sowie die gezielte Untersuchung von Patienten, bei denen der Verdacht besteht, unter einer psychischen Begleiterkrankung zu leiden [23]. Beide Ansätze schließen sich keineswegs gegenseitig aus.

So sollte ein Patient, der zwar ein Screening ohne Befund passiert, jedoch Anzeichen einer psychischen Begleiterkrankung aufweist, tiefergehend untersucht werden [23]. Zahlreiche internationale Leitlinien empfehlen ein frühes Screening auf psychische Begleiterkrankungen [19][23]. Dadurch kann eine rechtzeitige interdisziplinäre Diagnostik das Erkennen der Begleiterkrankung sowie eine zeitnahe Zielsetzung mit darauf abgestimmter Therapie ermöglichen [23]. 


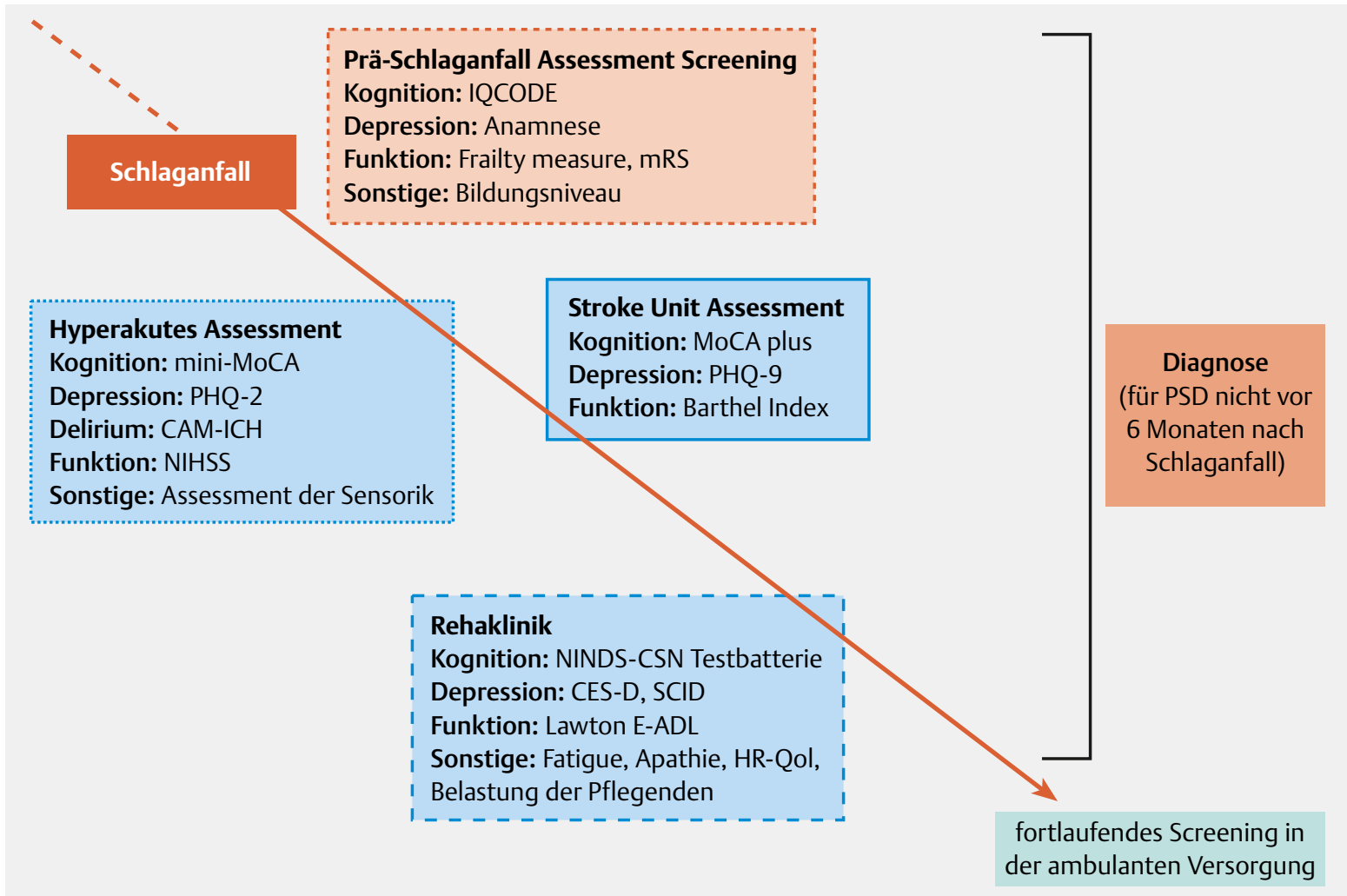

- Abb. 3 Neuropsychologische Assessments über den gesamten Krankheitsverlauf des Assessment Schlaganfalls hinweg. Schematische Illustration eines möglichen Ansatzes in verschiedenen Stadien. Die genannten Tests werden in der internationalen Literatur als Beispiele angeführt. Sie stellen jedoch keine Empfehlung dar und sind möglicherweise nicht auf Deutsch verfügbar. Beachtenswert ist, dass alle Domänen genutzt werden, um die klinische Diagnosefindung zu unterstützen (Kognition, Depression, Funktion, Sonstige). Kurz nach dem Ereignis wird das Augenmerk eher auf die Kognition vor dem Schlaganfall, ein Delirium und durch den Schlaganfall verursachte Einschränkungen gelegt als auf ein detailliertes kognitives Assessment. CAM-ICU: Confusion Assessment Method for the Intensive Care Unit, CES-D: Centre for Epidemiologic Studies Depression, E-ADL: Extended Assessment.

CAM-ICU: Confusion Method for Activities of Daily Living, HR-QoL: Health-Related Quality of Life, IQCODE: Informant Questionnaire for Cognitive Decline in the Elderly, MoCA: Montreal Cognitive Assessment (mini-MoCA: Kurzform des MoCA; MoCA plus: MoCA mit zusätzlichen Tests, wie von der European Stroke Organisation empfohlen), mRS: modified Rankin Scale, NIHSS: National Institutes Health Stroke Scale, NINDS-CSN: National Institute Neurological Disorders and Canadian Stroke Network, PHQ: Patient Health Questionnaire und SCID: Structured Clinical Interview Depression. (Quelle: Grafik nach Quinn et al. [23]; Umsetzung: Thieme Gruppe)

Assessments Ein Screening auf PSCI und PSD nach Schlaganfall wird jedoch nur selten vorgenommen, und wenn doch, werden dabei verschiedenste Assessments verwendet [12]. Eine systematische Übersichtsarbeit zeigte, dass von 8.826 Schlaganfallstudien, die zwischen den Jahren 2002 und 2011 publiziert und analysiert wurden, lediglich 488 (6\%) die kognitiven Folgen eines Schlaganfalls maßen und dabei 367 verschiedene Assessments verwendeten [12]. Neben dem Screening auf psychische Begleiterkrankungen nach Schlaganfall sollte jedoch stets auch eine Bewertung der Körperfunktionen, z. B. mit der modifizierten Rankin-Skala (mRS) oder dem Barthel-Index (BI), erfolgen, um ein vollständiges Bild der Einschränkungen zu erhalten [19][23].

Es gibt kein perfektes neuropsychologisches Assessment zur Erfassung der psychischen Begleiterkrankungen nach Schlaganfall. Der bevorzugt verwendete Test wird sich un- terscheiden hinsichtlich des Ziels der Testung, der Patientenklientel und der Fähigkeiten der Tester. Je nach Zeitpunkt und Setting der Untersuchung kommen andere Assessments zum Screening infrage. $\mathbf{A b b}$. $\mathbf{3}$ zeigt einen möglichen Ansatz zum Screening auf psychische Begleiterkrankungen nach Schlaganfall über den gesamten Krankheitsverlauf und über die Domänen Kognition, Depression und Funktion hinweg [23].

Auswahl des Assessments Eine systematische Übersichtsarbeit über die psychometrischen Eigenschaften von Assessments zu kognitiven Einschränkungen in verschiedenen Domänen nach Schlaganfall ergab, dass die am häufigsten benutzten Assessments eine vergleichbare Genauigkeit besitzen und sich kein Assessment gegenüber den anderen als deutlich überlegen zeigte [14]. Bei der Auswahl eines geeigneten Assessments sollten Verfügbarkeit, Vertrautheit mit ihm und Durchführbarkeit 
- Tab. 1 Diagnostische Testgenauigkeit von häufig in Studien zu PSCl verwendeten Assessments im Vergleich zum Referenzstandard (jegliches anerkannte Klassifikationssystem) [14]

\begin{tabular}{|c|c|c|c|c|c|c|}
\hline Test (Schwelle) & $\begin{array}{l}\text { Publikationen } \\
\text { (Patienten) }\end{array}$ & $\begin{array}{l}\text { Kognitive Ein- } \\
\text { schränkungen (\%) }\end{array}$ & $\begin{array}{l}\text { Sensitivität } \\
(95 \% \mathrm{Cl})\end{array}$ & $\begin{array}{l}\text { Spezifität } \\
(95 \% \mathrm{Cl})\end{array}$ & $\begin{array}{l}\text { Positive Likelihood } \\
\text { Ratio }(95 \% \mathrm{Cl})\end{array}$ & $\begin{array}{l}\text { Negative Likelihood } \\
\text { Ratio }(95 \% \mathrm{Cl})\end{array}$ \\
\hline ACE-R $(<88 / 100)$ & $2(192)$ & $52(27 \%)$ & $96,2(0,90 \ldots 1,0)$ & $0,70(0,59 \ldots 0,80)$ & $3,19(2,24 \ldots .4,54)$ & $0,06(0,01 \ldots 0,22)$ \\
\hline MMSE $(<25 / 30)$ & 12 (1639) & $483(30 \%)$ & $0,71(0,60 \ldots 0,80)$ & $0,85(0,80 \ldots 0,89)$ & $4,73(3,63 \ldots .6,17)$ & $0,34(0,25 \ldots 0,47)$ \\
\hline MMSE $(<27 / 30)$ & $5(445)$ & $195(44 \%)$ & $0,88(0,82 \ldots 0,92)$ & $0,62(0,50 \ldots 0,73)$ & $2,33(1,72 \ldots 3,17)$ & $0,19(0,13 \ldots 0,29)$ \\
\hline $\operatorname{MoCA}(<22 / 30)$ & $6(726)$ & $289(39 \%)$ & $0,84(0,76 \ldots 0,89)$ & $0,78(0,69 \ldots 0,84)$ & $3,75(2,77 \ldots .5,08)$ & $0,20(0,15 \ldots 0,29)$ \\
\hline $\operatorname{MoCA}(<26 / 30)$ & $4(326)$ & $131(40 \%)$ & $0,95(0,89 \ldots 0,98)$ & $0,45(0,34 \ldots 0,57)$ & $1,73(1,43 \ldots 2,10)$ & $0,10(0,04 \ldots 0,23)$ \\
\hline R-CAMCOG $(<33 / 49)$ & $2(421)$ & $90(21 \%)$ & $0,81(0,57 \ldots 0,93)$ & $0,92(0,87 \ldots 0,95)$ & $10,18(6,41 \ldots 16,18)$ & $0,20(0,07 \ldots 0,52)$ \\
\hline
\end{tabular}

einbezogen werden. Ein geeigneter Test könnte z. B. das Montreal Cognitive Assessment (MoCA) mit einem Schwellenwert für PSCI von weniger als 26 von 30 Punkten sein. Dieses Assessment ist kostenfrei auf Deutsch verfügbar und hochsensitiv, um möglicherweise beeinträchtigte Personen identifizieren zu können ( $\triangleright$ Tab. 1). Neuere Ansätze zum Screening auf PSCI stellen Biomarker in Form von bildgebenden Verfahren, metabolischen, genetischen und Entzündungsmediatoren dar [19]. Bislang war die Suche nach einem präzisen und verlässlichen biochemischen Indikator zwar noch nicht erfolgreich, jedoch ließ sich ein Zusammenhang zwischen erhöhter Blutsenkungsgeschwindigkeit und einem reduzierten Volumen des Hippocampus mit schlechterer kognitiver Leistung nach Schlaganfall zeigen $[3,9,18]$.

Symptome vor dem Schlaganfall Eine besondere Bedeutung kommt hierbei auch dem retrospektiven Testen auf Symptome einer kognitiven Einschränkung bereits vor dem Schlaganfall zu. Hierfür sollten Angehörige des Patienten z. B. mit dem Fragebogen zur geistigen Leistungsfähigkeit für ältere Personen (IQCODE) befragt werden [23]. Der Fragebogen ist ins Deutsche übersetzt und im Internet frei verfügbar.

Sechs-Monats-Zeitraum Die meisten Klassifikationen definieren PSCl als eine kognitive Einschränkung, die innerhalb von sechs Monaten nach dem Schlaganfall beginnt und sich nicht zurückbildet [25]. Daraus kann gefolgert werden, dass die Diagnose PSCl erst nach einem Zeitraum von sechs Monaten sichergestellt werden kann und das klinische Assessment bis dahin über einen längeren Zeitraum erfolgen sollte [23].

Am häufigsten verwendete Assessments Die am häufigsten verwendeten Assessments in Studien zu den psychischen Begleiterkrankungen nach Schlaganfall sind die Hamilton Rating Scale Depression (HRSD), Short Form-36 Health Survey (SF-36), Hospital Anxiety and Depression Scale (HADS-D), Beck-Depressions-Inventar Fast Screen (BDI-FS) und die Geriatrische Depressionsskala (GDS) [12]. Alle oben genannten Skalen liegen auch auf Deutsch vor.
Delir Für das Assessment des Delirs im hyperakuten Setting ist die CAM-ICU-Skala geeignet, die auch auf Deutsch und kostenfrei im Internet verfügbar ist [2]. Eine weitere mögliche Skala, die auf das Vorliegen eines Delirs sowie auf kognitive Einschränkungen testet, ist die 4A-Skala, die kostenfrei und auf Deutsch im Internet verfügbar ist [13]. Sie steht ihrem Referenzstandard CAM-ICU in nichts nach [4]. Bei der täglichen Delir-Surveillance sollte aufgrund von Übungseffekten Frage 3 jedoch ausgelassen werden [15].

Terminologie des DSM-5 Die weiter oben aufgeführten Assessments zum Screening auf psychische Begleiterkrankungen nach Schlaganfall sind eher nicht dazu geeignet, eine klinische Diagnose auf PSCI oder PSD im engeren Sinne zu stellen [23]. Dafür braucht es z. B. die Terminologie des DSM-5, des diagnostischen und statistischen Leitfadens psychischer Störungen (Diagnostic and Statistical Manual of Mental Disorders), die auch auf Menschen nach Schlaganfall anwendbar ist [23]. Das Kriterium, anhand dessen das DSM-5 eine leichte von einer schweren neurokognitiven Einschränkung unterscheidet, ist der Verlust der Aktivitäten des täglichen Lebens (ADL) [23].

\section{Prognose}

Eine psychische Begleiterkrankung nach Schlaganfall führt zu einer schlechteren Prognose.

Eine prospektive Kohortenstudie mit 324 Teilnehmern zeigte, dass Menschen nach Schlaganfall, die drei Monate nach dem Ereignis unter einer PSCI litten, ein 6,3-fach erhöhtes Risiko hatten, innerhalb von zwei Jahren nach dem Ereignis zu versterben - im Vergleich zu Erkrankten ohne PSCI (relatives Risiko (RR) =6,3; $95 \%$ Konfidenzintervall (KI) 2,3...17,3) [1]. Das Risiko war umso höher, je weniger Anzeichen einer kognitiven Einschränkung die Patienten vor dem Schlaganfall aufwiesen.

Ein möglicher Erklärungsansatz für die erhöhte Mortalität ist, dass kognitiv eingeschränkte Menschen nach Schlaganfall eine geringere Compliance bei der Anwendung von Therapien zeigen. Dies wiederum resultiert in einer weniger effektiven Prophylaxe eines Reinfarktes und führt 
somit zu einem erhöhten Risiko für einen Reinfarkt und Tod. Ein weiterer möglicher Grund wäre, dass kognitive Einschränkungen eher bei bereits geschwächten Individuen auftreten, sodass deren Schwäche selbst bereits eine schädliche Erkrankung darstellt, die die Mortalität weiter erhöht [1].

In einer Metaanalyse von 23 Beobachtungsstudien mit 18374 Teilnehmern zeigte sich, dass Depressionen nach Schlaganfall konsistent häufiger auftraten, wenn bereits vor dem Schlaganfall depressive Symptome vorlagen oder sich ein schwerwiegenderes neurologisches Defizit sowie bedeutendere körperliche Einschränkungen in der Akutphase zeigten [11]. Dies wiederum löst einen Teufelskreis aus: Patienten mit größeren funktionellen Einschränkungen entwickeln eher eine Depression, die wiederum das Therapieergebnis dieser besonders schwer betroffenen Patienten negativ beeinflusst. Es zeigte sich jedoch kein konsistenter Zusammenhang zwischen Depression und kognitiven Einschränkungen nach Schlaganfall [11].

Höheres Lebensalter, Sehstörungen, eine Infektion der Harn- oder Atemwege und bestehende kognitive Vorerkrankungen sind üblicherweise Risikofaktoren für ein Delir [8][24]. Ein Delir kann zwar ohne Folgen für die Patienten bleiben, jedoch auch zu einem längeren Krankenhausaufenthalt führen, der mit einer höheren Mortalität verbunden ist. Längerfristige Folgen sind kognitive Verschlechterung sowie funktionelle Defizite und Abhängigkeit von fremder Hilfe [8].

\section{Behandlungsansätze}

\section{Körperliche Aktivität}

Körperliche Aktivität verbessert die Kognition nach Schlaganfall und kann helfen, die Entstehung einer Depression oder eines Delirs zu verhindern. Die sicherlich effektivste Art, das Auftreten einer PSCI zu unterbinden, besteht in optimaler Akutbehandlung und intensiver Sekundärprävention [19]. Dies umfasst multifaktorielle Interventionen wie eine mediterrane Diät, moderater Alkoholkonsum und Nikotinentwöhnung genauso wie die Gabe von Antikoagulanzien, Antihypertensiva und Statinen, aber auch kognitives Training sowie körperliche Aktivität.

In einer systematischen Übersichtsarbeit zur Wirkung von körperlicher Aktivität auf die kognitive Funktion nach Schlaganfall mit 14 randomisierten kontrollierten Studien mit 736 Teilnehmern zeigte sich insgesamt ein positiver Effekt auf die kognitive Leistung (standardisierte Mittelwertdifferenz, SMD = 0,3; $95 \%$ KI 0,14...0,47) [20]. Hierbei zeigten sich die größten Veränderungen im Mittel mehr als zwei Jahre nach dem Ereignis in der chronischen Phase. Körperliche Aktivität scheint insbesondere die Aufmerksamkeit/Verarbeitungsgeschwindigkeit zu fördern, wohingegen die exekutiven Funktionen und das Arbeitsgedächtnis sich nicht signifikant zu verbessern scheinen [20].

Übungsprogramm Ein kombiniertes Übungsprogramm aus sowohl Kraft- und Balancetraining als auch Ausdauertraining zeigte die größten Effekte. Die komplexe therapeutische Intervention der kognitiven Rehabilitation zielt darauf ab, die kognitive Funktion nach einem Schlaganfall zu verbessern. Sie wird üblicherweise als Einzelbehandlung durch Neuropsychologen, als Gruppentraining und kognitives Computertraining angeboten. Sie stellt einen integralen Bestandteil der multidisziplinären Schlaganfallrehabilitation dar und sollte unmittelbar nach Eintreten des Schlaganfalls beginnen. Sie hat sich in einer systematischen Cochrane-Übersichtsarbeit mit 13 randomisierten kontrollierten Studien mit 514 Teilnehmern zur Verbesserung der selbst eingeschätzten Gedächtnisleistung in der postakuten Phase nach Schlaganfall als effektiv erwiesen (SMD = 0,3; $95 \% \mathrm{KI}(0,08 \ldots 0,64)$, moderate Qualität der Evidenz) [5]. Diese Effekte waren jedoch mehr als drei Monate nach Ende der Intervention nicht mehr nachweisbar (SMD = 0,31; $95 \%$ KI -0,02...0,64; niedrige Qualität der Evidenz).

\section{Nichtinvasive Hirnstimulation}

Ein innovativer Ansatz zur Behandlung von PSCI stellt die nichtinvasive Hirnstimulation mittels transkranieller Gleichstromstimulation (transcranial Direct Current Stimulation, tDCS) sowie repetitiver transkranieller Magnetstimulation (repetitive Transcranial Magnetic Stimulation, rTMS) dar. Allerdings fehlen hierzu bislang aussagekräftige Studien mit adäquaten Stichprobengrößen, einem optimalen Stimulationsprotokoll und adäquater Berücksichtigung der individuellen effektmodulierenden Einflussvariablen, wie z. B. die Schädelform der Patienten oder deren genetische Polymorphismen.

\section{Pharmakotherapie}

In einer systematischen Cochrane-Übersichtsarbeit mit 16 randomisierten kontrollierten Studien und 1655 Teilnehmern über die Effekte von Interventionen zur Behandlung von PSD zeigte sich, dass Pharmakotherapie sowohl eine signifikante Verbesserung der Symptome als auch einen signifikanten Anstieg an unerwünschten Nebenwirkungen verursachte [6]. Es zeigten sich jedoch keine Hinweise auf die Wirksamkeit von Psychotherapie [6]. Der weiter oben bereits erwähnte Mangel an einheitlichen Assessments für die Schwere der Depression erschwerte die Auswertung.

Ein weiterer, indirekter Ansatz zielt ab auf die Beseitigung der Einschränkung der Körperfunktionen als Risikofaktor für eine Depression nach Schlaganfall durch eine frühzeitige und effektive Therapie [11]. Eine häufige Komorbidität von Depressionen, insbesondere nach linkshemisphärischem Schlaganfall, sind Angststörungen, die speziell bei rechtshemisphärischem Schlaganfall auch isoliert auftreten können [4]. In einer systematischen Cochrane-Über- 
sichtsarbeit mit drei randomisierten kontrollierten Studien und 196 Teilnehmern zeigte sich, dass es bisher keine überzeugenden Untersuchungen zur Effektivität verschiedener Interventionen, wie z. B. Pharmakotherapie mittels Paroxetin oder Buspiron sowie Psychotherapie und Entspannungsübungen, gibt (sehr niedrige Qualität der Evidenz) [10].

Die Therapie des Delirs ist symptomatisch und umfasst medikamentöse und nichtmedikamentöse Ansätze [8]. Der medikamentöse Ansatz umfasst die Gabe von niedrig dosierten Antipsychotika. Der nichtmedikamentöse Ansatz umfasst die Beseitigung von Risiko- und auslösenden Faktoren des Delirs und scheint effektiver zur Prävention eines Delirs zu sein als zu dessen Therapie [8].

\section{Ausblick}

Künftig sind ein regelmäßiges Screening und eine frühzeitige, effektive Therapie gefordert.

Psychische Begleiterkrankungen nach Schlaganfall sind ein hochkomplexes Thema: Konzepte überlappen sich, und es gibt keine trennscharfen Definitionen. Menschen nach Schlaganfall werden selten routinemäßig auf das Vorliegen von psychischen Begleiterkrankungen hin getestet, unterschiedliche Messinstrumente erschweren die Vergleichbarkeit der Daten.

Ein wünschenswerter Ansatz wäre, alle Menschen nach Schlaganfall mit einem geeigneten Assessment, wie z. B. dem MoCA mit dem Schwellenwert 26 von 30 Punkten für PSCI und z. B. der HADS-D für PSD und dem 4A-Test für Delir, auf das Vorliegen von psychischen Begleiterkrankungen hin zu untersuchen. Patienten mit einem positiven Screening würden dann in einem nächsten Schritt von Spezialisten tiefergehend untersucht, und zwar hinsichtlich kognitiver und funktionaler Einschränkungen. Die effektive Therapie eingeschränkter Körperfunktionen sollte unmittelbar beginnen, um einen Abbau der kognitiven Leistungsfähigkeit und die Entstehung einer Depression zu verhindern.

Die Ergebnisse der Assessments zu PSCI und PSD stellen keine klinischen Diagnosen im engeren Sinne dar. Die Di- agnosestellung erfolgt ausschließlich über ICD-10 oder DSM-5. Die Diagnose einer PSCI sollte frühestens sechs Monate nach dem Schlaganfall gestellt werden, da sich deren Symptome bis dahin häufig zurückbilden können.

\section{Autorinnen/Autoren}

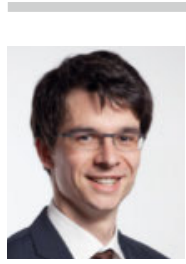

\section{Prof. Dr. Bernhard Elsner}

Prof. Dr. Bernhard Elsner ist Physiotherapeut und Gesundheitswissenschaftler. Er ist Professor für Therapiewissenschaften und Studiengangsleiter der Studiengänge Physiotherapie und Ergotherapie an der SRH Hochschule für Gesundheit in Gera.

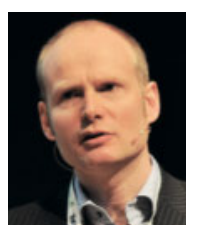

\section{Prof. Dr. Jan Mehrholz}

Prof. Dr. Jan Mehrholz ist Physiotherapeut und Professor für Therapiewissenschaften. Zudem leitet er das Wissenschaftliche Institut der Klinik Bavaria in Kreischa und ist als Privatdozent für Gesundheitswissenschaften/Public Health an der Medizinischen Fakultät der TU Dresden tätig.

\section{Korrespondenzadresse}

Prof. Dr. Bernhard Elsner, MPH

Studiengangsleiter Physiotherapie/Ergotherapie

SRH Hochschule für Gesundheit

Neue Str. 28-30

07548 Gera

E-Mail: bernhard.elsner@srh.de

\section{Literatur}

Die komplette Literaturliste finden Sie unter www.thieme-connect.de/products

Bibliografie

DOI https://doi.org/10.1055/a-0596-6456

neuroreha 2018; 10: 71-76

(c) Georg Thieme Verlag KG Stuttgart · New York ISSN 1611-6496 